\title{
Controle de doenças do trigo com fosfitos e acibenzolar-s-metil isoladamente ou associados a piraclostrobina + epoxiconazole
}

\section{Control of wheat diseases using phosphites and acibenzolar-s-methyl alone or associated with piraclostrobina + epoxiconazole}

\author{
Hellen Aparecida Arantes dos Santos ${ }^{1 *}$; Maristella Dalla Pria ${ }^{2}$; Olavo Corrêa da \\ Silva $^{3}$; Louise Larissa May De Mio ${ }^{4}$
}

\begin{abstract}
Resumo
Foi realizado um experimento no município de Palmeira-PR, visando avaliar o efeito de diferentes fontes de fosfitos no controle da ferrugem da folha, do oídio e das manchas amarela e marrom na cultura do trigo. Os tratamentos foram: quatro fontes comerciais de fosfitos (A, B, C e D) aplicados na dose de $1500 \mathrm{~mL} \mathrm{ha}^{-1}$ (dose de produto ativo) isolado ou associado aos demais produtos, acibenzolarS-metil (ASM) na dose de $500 \mathrm{~mL} \mathrm{ha}^{-1}$ (isolado ou associado), piraclostrobina + epoxiconazole (fungicida) na dose de $500 \mathrm{~mL} \mathrm{ha}^{-1}$, e as combinações de fosfito A com piraclostrobina + epoxiconazole na dose de $300 \mathrm{~mL} \mathrm{ha}^{-1}$, fosfito A com piraclostrobina + epoxiconazole na dose de $400 \mathrm{~mL} \mathrm{ha}^{-1}$, ASM com piraclostrobina + epoxiconazole na dose de $400 \mathrm{~mL} \mathrm{ha}^{-1}$ e testemunha sem controle. Utilizouse delineamento em blocos ao acaso, com quatro repetições. No caso das doenças, as avaliações consistiram na determinação da severidade e cálculo de área abaixo da curva de progresso da doença em folha bandeira e em planta inteira. Foram avaliados também: índice de área foliar, peso de mil sementes, peso hectolítrico e produtividade. Fosfito, independente das fontes testadas, não apresentou ação sobre ferrugem da folha, oídio, e manchas amarela ou marrom do trigo. O indutor de resistência acibenzolarS-metil isoladamente ou em associação com fungicidas afetou apenas a ferrugem da folha e o oídio do trigo. Apenas os fosfitos associados ao fungicida controlaram as doenças, mas não houve aumento adicional de produtividade quando comparado com o controle proporcionado somente pelo fungicida.

Palavras-chave: Fosfitos. Drechslera tritici-repentis. Bipolaris sorokiniana. Puccinia triticina. Blumeria graminis. Produtividade.
\end{abstract}

\begin{abstract}
The objective of this study was to evaluate the effect of different sources of phosphite to control leaf rust, powdery mildew, yellow and brown spot in the wheat crop in Palmeira, Paraná state, Brazil. The treatments were: four commercial sources of phosphites (A, B, C and D), applied at $1500 \mathrm{~mL} \mathrm{ha}^{-1}$, (applied in rate of active product) acibenzolar-S-methyl (ASM) at $500 \mathrm{~mL} \mathrm{ha}^{-1}$ (isolad or association), pyraclostrobin + epoxiconazole (fungicide) at $400 \mathrm{~mL} \mathrm{ha}^{-1}$ ) and phosphite A with pyraclostrobin + epoxiconazole (at $\left.300 \mathrm{~mL} \mathrm{ha}^{-1}\right)$, phosphite A with pyraclostrobin + epoxiconazole $\left(400 \mathrm{~mL} \mathrm{ha}^{-1}\right)$, acibenzolar-S-methyl with pyraclostrobin + epoxiconazole $\left(400 \mathrm{~mL} \mathrm{ha}^{-1}\right)$ and control (water), . The
\end{abstract}

\footnotetext{
1 Eng $^{\mathrm{o}}$ Agr $^{\mathrm{o}}$, Mestre em Agronomia, Prof ${ }^{\mathrm{a}}$ do Curso de Ciências Rurais, UFSC, Rodovia Municipal Ulisses Gaboardi, Km 3, Fazenda Pessegueirinho, 89520-000,Curitibanos, SC, E-mail: hellen_harantes@yahoo.com.br

2 Eng $^{\mathrm{o}} \mathrm{Agr}^{\mathrm{o}}$, Pós Doutora em Fitopatologia, Prof ${ }^{\mathrm{a}}$ do Departamento de Fitotecnia e Fitossanidade, Universidade Estadual de Ponta Grossa, UEPG, Av. Gal. Carlos de Cavalcanti, 47488, 84030-900, Ponta Grossa, PR, E-mail: mdallapria@uol.com.br

3 Eng $^{\circ}$ Agr $^{\circ}$, Mestre em Crop Science, Doutorando em Produção Vegetal, Departamento de Fitotecnia e Fitossanitarismo, Universidade Federal do Paraná, UFPR. Rua dos Funcionários 1540, 80035-050, Curitiba, PR, E-mail:OCorrea@dow.com

4 Eng $^{\mathrm{o}}$ Agr $^{\mathrm{o}}, \mathrm{Dr}^{\mathrm{a}}$ em Fitopatologia, Prof ${ }^{\mathrm{a}}$ do Departamento de Fitotecnia e Fitossanitarismo, Universidade Federal do Paraná, UFPR., Curitiba, PR, E-mail: maydemio@ufpr.br

* Autor para correspondência
}

Recebido para publicação 09/09/2009 Aprovado em 14/03/201 1 
experimental design adopted was complete randomized blocks with four replications. Regarding diseases, evaluations were done on the determination of severity and the calculation of the area below the curve of the disease progress in the flag leaf and entire plant. Leaf area index, thousand seeds weight, hectoliter weight and yield were also evaluated. Phosphite, independently source, did not act on leaf rust, powdery mildew, yellow and brown spot. The resistance inducer acibenzolar-S-methyl either isolated or associated to other fungicides affected leaf rust and powdery mildew of the wheat. Only phosphites associated to the fungicide controlled diseases, however there was no additional increase in yield whether compared to the control promoted by the fungicide.

Key words: Phosphites. Drechslera tritici-repentis. Bipolaris sorokiniana. Puccinia triticina. Blumeria graminis. Yield.

\section{Introdução}

Na região dos Campos Gerais a cultura do trigo tem grande importância na produção de grãos, no entanto seu rendimento pode ser comprometido pelo aparecimento do complexo de doenças foliares que ocorrem durante todo ciclo.

O oídio causado por Erysiphe graminis DC. F. sp. tritici Em. Marchal Erysiphe graminis DC. = Blumeria graminis (DC.) E.O. Speer ocorre de forma endêmica em áreas tritícolas de clima frio e úmido no Brasil, especialmente na Região Sul e em lavouras sob sistema irrigado nas Regiões CentroOeste e Sudeste (REIS; CASA, 2007).

A ferrugem da folha do trigo (Puccinia triticina Erikss) ocorre em praticamente todas as regiões tritícolas do país. Os danos decorrentes de epidemias de P. triticina, vão depender do estádio fenológico da planta, da suscetibilidade da cultivar, da virulência da raça fisiológica e das condições ambientais (REIS; CASA, 2007). Em cultivares suscetíveis já foram relatadas perdas de até $50 \%$ no rendimento dos grãos (REIS; CASA; FORCELINI, 1996).

A mancha marrom (Bipolaris sorokiniana c. In Sorok.) e a mancha amarela (Dreschlera triticirepentis Died.) são consideradas as doenças mais prejudiciais para a cultura do trigo (MEHTA et al., 1992), podendo acarretar perdas de 20 a $80 \%$ no rendimento da cultura (MEHTA, 1993; HETZLER et al., 1991; BARROS, 1985). Entre as manchas foliares, a mancha amarela é a que ocorre com maior frequência em plantio direto, sendo responsável pelas epidemias mais agressivas de manchas foliares (REIS; CASA; MEDEIROS, 2001).
A adoção de medidas conjuntas, como rotação de culturas, eliminação de plantas voluntárias e de hospedeiros secundários, tratamento e utilização de sementes sadias, uso de cultivares resistentes, aplicação de fungicidas e de produtos alternativos (REIS; CASA, 2007), como os fosfitos, torna mais eficiente o controle das doenças do trigo.

Os fosfitos são derivados da neutralização do ácido fosforoso $\left(\mathrm{H}_{3} \mathrm{PO}_{3}\right)$ por uma base que pode ser hidróxido de sódio, hidróxido de amônio ou hidróxido de potássio, entre outros, sendo este último o mais utilizado formando o fosfito de potássio (REUVENI, 1997).

As principais vantagens apresentadas pelo uso dos fosfitos são: rápida absorção do produto pelas plantas, assimilação total (diferentemente dos fosfatos), exigência de menos energia da planta, favorecimento da absorção de $\mathrm{Ca}, \mathrm{B}, \mathrm{Zn}, \mathrm{Mn}$, Mo, $\mathrm{K}$ e outros elementos (por ser um excelente complexante), controle e prevenção de doenças fúngicas (VITTI et al., 2005), além da possibilidade de atuarem como ativadores de resistência das plantas por meio do estímulo da produção de algumas fitoalexinas (GUEST; GRANT, 1991; JACKSON et al., 2000).

Existem poucos trabalhos publicados referidos à utilização de marcas comerciais de fosfito, os quais apontam resultados positivos para a cultura do tomate no controle de doenças (NASCIMENTO et al., 2008). A maioria dos relatos que se referem ao uso de fosfitos de potássio tratam do uso deste isolado ou associado a outros produtos, como nos trabalhos de Moreira et al. (2002) e Sônego, Garrido 
e Czermainski (2003) que mostram eficiência no controle de podridão parda em pós colheita em fruteiras de caroço e em míldio da videira, tanto em folha quanto no cacho. Isso demonstra o desconhecimento sobre o efeito do uso de marcas comerciais de fosfitos para cereais de inverno, como o trigo. Desta forma, este trabalho objetivou avaliar o controle de oídio, da ferrugem da folha, da mancha marrom e da mancha amarela, por meio do uso de diferentes formulações comerciais de fosfitos, acibenzolar-S-metil (ASM) e das combinações de fosfito com ASM e com piraclostrobina + epoxiconazole (em duas doses), além de estimar variáveis relacionadas à produtividade na cultura do trigo.

\section{Materiais e Métodos}

O experimento foi instalado na Fazenda Santa Carlota no município de Palmeira-PR, localizada nas coordenadas de $25^{\circ} 37^{\prime}$ de latitude Sul, longitude de $50^{\circ} 21^{\prime}$ 'Oeste e altitude de $820 \mathrm{~m}$, durante a safra de 2006.

$\mathrm{Na}$ área em que foi instalado o experimento predomina o solo classificado como PODZÓLICO VERMELHO-AMARELO, textura média, fase campo subtropical e relevo suave ondulado (EMBRAPA, 1999a).

Três dias antes da semeadura do trigo, a área foi dessecada com aplicação de glifosato (480 g i.a. $\left.\mathrm{L}^{-1}\right)$. As sementes (cultivar Avante) foram previamente tratadas com imidacloprido (60 g i.a. $100 \mathrm{~kg}^{-1}$ de sementes). A semeadura do trigo foi realizada no dia 07 de julho de 2006, pelo método de semeadura direta sobre a palhada da cultura do milho, com espaçamento entre linhas de $0,17 \mathrm{~m}$ e 230 plantas por metro quadrado.

A adubação de base foi realizada antes da semeadura com $330 \mathrm{~kg} \mathrm{ha}^{-1}$ de adubo NPK da fórmula comercial 14-34-00. A adubação em cobertura foi realizada durante o perfilhamento com $260 \mathrm{~kg} \mathrm{ha}^{-1}$ de NPK da fórmula comercial 25-00-25.
Durante a fase de elongação e de floração foram realizadas aplicações do inseticida lufenuron $(5 \mathrm{~g}$ i.a. $\mathrm{L}^{-1}$ ) para controle da lagarta militar (Spodoptera frugiperda) e lagarta do trigo (Pseudaletia sequax Franclemont).

As avaliações de severidade foram realizadas semanalmente, a partir da antese completa até a formação do grão, em dez folhas bandeiras e em dez plantas inteiras, totalizando cinco avaliações. Para tanto foram atribuídas porcentagens da área foliar lesionada. A área abaixo da curva de progresso da doença (AACPD) foi calculada com os dados de severidade de folha bandeira e de planta inteira, pela equação proposta por Shaner e Finny (1977) descrita abaixo.

n-1

$$
\left.\mathrm{AACPD}=\sum[(\mathrm{yi}+\mathrm{yi}+1) / 2) \times(\mathrm{ti}+1-\mathrm{ti})\right]
$$

Onde: n é o número de avaliações;

y a intensidade de doenças;

t o tempo quando da avaliação da intensidade da doença;

i e i+1 representam as observações de $1 \mathrm{a}$.

Foram realizadas avaliações dos teores de nitrogênio $(\mathrm{N})$, fósforo $(\mathrm{P})$, potássio $(\mathrm{K})$ e manganês (Mn) presentes nas folhas. Na determinação dos teores de N, P, $\mathrm{K}$ e Mn, foram coletadas 40 folhas bandeira por parcela conforme metodologia descrita por Raij et al. (1997), durante a antese (antese completa). As análises foram realizadas no Laboratório de análises Físico-químicas da Fundação $\mathrm{ABC}$, localizado em Castro. Para digestão de $\mathrm{N}$ total foi utilizado o método de combustão de Dumas em analisador Leco $^{\circledR}$ FP-428 e para os demais elementos foi realizada a digestão Nítricoperclórica (EMBRAPA, 1999b).

$\mathrm{O}$ índice de área foliar (IAF) foi mensurado com o auxílio do medidor de radiação solar fotossinteticamente ativa (accuPAR) Ceptomero Linear, realizando duas medições a $\pm 1 \mathrm{~m}$ acima do solo e quatro a $\pm 0,1 \mathrm{~m}$ sobre o solo, durante o 
período de formação do grão.

Os tratamentos utilizados são descritos na (Tabela 1). O delineamento experimental foi em blocos casualizados com quatro repetições. Cada unidade experimental foi composta por parcelas de 2,38 x 5,0 metros, contendo 14 linhas de semeadura. Os tratamentos foram aplicados com auxílio de pulverizador costal, à pressão constante de $131 \mathrm{kPa}$, pelo dióxido de carbono comprimido, equipado com 6 pontas de jato "plano" XR11002 VS com espaçamento de $0,5 \mathrm{~m}$, utilizando um volume de calda de $135 \mathrm{~L} \mathrm{ha}^{-1}$. Os produtos foram aplicados duas vezes, sendo uma aplicação no estádio 45 e outra no estádio 60 da escala de Zadoks, Chang e Konzak (1974).

Tabela 1. Tratamentos utilizados para controle do oídio (Blumeria graminis f. sp. tritici), ferrugem das folhas (Puccinia trticina), mancha marrom (Bipolaris sorokiniana) e mancha amarela (Dreschlera triticirepentis) com ingrediente ativo, classe e doses de ingrediente ativo (g i.a. $\mathrm{L}^{-1}$ ) e dose do produto comercial

\begin{tabular}{|c|c|c|c|c|}
\hline Tratamentos & Produto Comercial & Ingrediente ativo & Classe & Dose mL p.a. ha ${ }^{-1}$ \\
\hline Testemunha & Testemunha & ..... & ............... & ................ \\
\hline Fosfito A & Phytus K & $\mathrm{P}_{2} \mathrm{O}_{5}+\mathrm{K}_{2} \mathrm{O}$ & Ácido fosforoso & 1500 \\
\hline Fosfito B & Reforce & $\mathrm{P}_{2} \mathrm{O}_{5}+\mathrm{K}_{2} \mathrm{O}$ & Ácido fosforoso & 1500 \\
\hline Fosfito C & Starphós & $\mathrm{P}_{2} \mathrm{O}_{5}+\mathrm{K}_{2} \mathrm{O}$ & Ácido fosforoso & 1500 \\
\hline Fosfito D & Phytogard Mn & $\mathrm{P}_{2} \mathrm{O}_{5}+\mathrm{Mn}$ & Ácido fosforoso & 1500 \\
\hline ASM & Bion (ASM1) & acibenzolar-S-metil & Ativador de plantas & 50 \\
\hline Fungicida + & Opera + Phytus K & $\left(\right.$ pirac. + epox. $\left.{ }^{2}\right)+$ & Fun. Sistêmico + & \\
\hline Fosfito A & & $\mathrm{P}_{2} \mathrm{O}_{5}+\mathrm{K}_{2} \mathrm{O}$ & Ac. Fosforoso & $300+1500$ \\
\hline Fungicida + & Opera + Phytus K & $($ pirac. + epox. $)+$ & Fun. Sistêmico + & $400+1500$ \\
\hline Fosfito A & & $\mathrm{P}_{2} \mathrm{O}_{5}+\mathrm{K}_{2} \mathrm{O}$ & Ac. Fosforoso & \\
\hline Fungicida + ASM & Opera + Bion & $($ pirac. + epox. $)+$ & Fun. Sistêmico + & $400+50$ \\
\hline & & ASM) & Ativador de plantas & \\
\hline Fungicida & Opera & $\begin{array}{l}\text { piraclostrobina }+ \\
\text { epoxiconazole }\end{array}$ & Fungicida sistêmico & 500 \\
\hline
\end{tabular}

${ }^{1} \mathrm{ASM}$ corresponde a acibenzolar-S-metil.

${ }^{2}$ pirac. + epox. corresponde a piraclostrobina + epoxiconazole.

A colheita foi realizada oito de novembro (42 dias após a antese), colhendo-se, de forma manual, nove linhas de quatro metros $\left(6,8 \mathrm{~m}^{2}\right)$. O rendimento de grãos foi expresso em $\mathrm{kg} \mathrm{ha}^{-1}$, com teor de umidade padronizado para $13 \%$, o peso de mil sementes foi determinado através da contagem e determinação da massa de 250 sementes e o peso hectolítrico (PH) foi obtido com o auxilio da balança Dalle Molle.

Para fins de análise, os dados de porcentagem de severidade foram transformados em arc sen $\sqrt{ } \mathrm{x} / 100$ (BANZATTO; KRONKA, 2006) e posteriormente submetidos à análise de variância pelo teste F. A comparação das médias, foi realizada pelo teste de Tukey a $5 \%$ de probabilidade.

\section{Resultados e Discussão}

Nas (Tabelas 2 e 3) são apresentados os dados de área abaixo da curva de progresso de manchas foliares, ferrugem e oídio em folha bandeira e em planta inteira respectivamente. Os formulados de fosfito, de modo geral não tiveram efeito sobre o 
controle das doenças foliares do trigo. Observase que a área abaixo da curva de progresso das manchas foliares em folha bandeira foi menor nos tratamentos com epoxiconazole + piraclostrobina de forma isolada ou associada ao fosfito A e ao ASM. Esses tratamentos também proporcionaram reduções nas áreas abaixo da curva de progresso da ferrugem e do oídio, em folha bandeira (Tabela 2) e planta inteira (Tabela 3). Costa, Zambolim e Rodrigues (2007) encontraram resultados semelhantes quando testaram ASM misturado ao fungicida piraclostrobina e azoxystrobin. Os mesmos autores verificaram controle eficiente da ferrugem do cafeeiro (Hemileia vastatrix Berk. \&
$\mathrm{Br}$ ), provavelmente devido ao efeito da estrobilurina presente no fungicida, do que do próprio efeito ASM. Esses resultados coincidem com os de outros pesquisadores que demonstram que as estrobilurinas controlam efetivamente alguns fungos pertencentes à divisão Basidiomycota (MARGOT et al., 1998). Outros autores também verificaram controle efetivo de doenças foliares com o uso de fungicidas do grupo químico das estrobilurinas. Bizi et al. (2008) testando produtos alternativos e fungicidas obtiveram $92 \%$ de controle de oídio em mudas de eucalipto, Dallagnol et al. (2006) obtiveram controle efetivo das manchas foliares do arroz irrigado com o uso de estrobilurinas.

Tabela 2. Área abaixo da curva de progresso das manchas foliares (Bipolaris sorokiniana e Drechslera tritici-repentis), ferrugem das folhas (Puccinia triticina) e oídio (Blumeria graminis f. sp. tritici) na folha bandeira após aplicação de diferentes fontes de fosfitos e de indutor de resistência isolados e associados a fungicida, na cultura do trigo, cultivar Avante. Safra 2006. Palmeira, PR.

\begin{tabular}{lllll}
\hline Tratamentos & Dose (mL p.c. ha-1) & Manchas Foliares & Ferrugem & Oídio \\
\hline Testemunha & $\ldots \ldots \ldots \ldots$ & $92,9 \mathrm{~b}^{1}$ & $750,1 \mathrm{~b}$ & $190,5 \mathrm{~b}$ \\
Fosfito A & 1500 & $75,8 \mathrm{~b}$ & $630,7 \mathrm{~b}$ & $208,9 \mathrm{~b}$ \\
Fosfito B & 1500 & $93,1 \mathrm{~b}$ & $762,7 \mathrm{~b}$ & $247,6 \mathrm{~b}$ \\
Fosfito C & 1500 & $83,9 \mathrm{~b}$ & $677,6 \mathrm{~b}$ & $178,2 \mathrm{~b}$ \\
Fosfito D & 1500 & $70,0 \mathrm{~b}$ & $667,2 \mathrm{~b}$ & $168,7 \mathrm{~b}$ \\
ASM & & $82,0 \mathrm{~b}$ & $521,7 \mathrm{~b}$ & $91,1 \mathrm{~b}$ \\
Fungicida + Fosfito A & $300+1500$ & $32,23 \mathrm{a}$ & $46,4 \mathrm{a}$ & $14,1 \mathrm{a}$ \\
Fungicida + Fosfito A & $400+1500$ & $42,3 \mathrm{a}$ & $15,1 \mathrm{a}$ & $8,7 \mathrm{a}$ \\
Fungicida + ASM & $400+50$ & $49,1 \mathrm{a}$ & $22,3 \mathrm{a}$ & $7,5 \mathrm{a}$ \\
Fungicida & 500 & $33,2 \mathrm{a}$ & $13,8 \mathrm{a}$ & $5,3 \mathrm{a}$ \\
C.V.(\%) & & 10,46 & 12,48 & 15,49
\end{tabular}

${ }^{1}$ Médias seguidas de letras distintas na mesma coluna diferem estatisticamente entre si pelo teste de Scott Knott a 5\% de probabilidade.

${ }^{2}$ ASM corresponde a acibenzolar-S-metil.

C.V.: Coeficiente de variação. 
Tabela 3. Área abaixo da curva de progresso das manchas foliares (Bipolaris sorokiniana e Drechslera tritici-repentis), ferrugem das folhas (Puccinia triticina) e oídio (Blumeria graminis f. sp. tritici) na planta inteira após aplicação de diferentes fontes de fosfitos e de indutor de resistência isolados e associados a fungicida, na cultura do trigo, cultivar Avante. Safra 2006. Palmeira, PR.

\begin{tabular}{|c|c|c|c|c|}
\hline Tratamentos & Dose (mL p.a. ha ${ }^{-1}$ ) & Manchas Foliares & Ferrugem & Oídio \\
\hline Testemunha & .............. & $100,5 \mathrm{a}^{1}$ & $784,5 \mathrm{~b}$ & $186,0 \mathrm{~b}$ \\
\hline Fosfito A & 1500 & $83,0 \mathrm{a}$ & $730,5 \mathrm{~b}$ & $227,4 \mathrm{~b}$ \\
\hline Fosfito B & 1500 & 97,9 a & $793,4 \mathrm{~b}$ & $263,4 b$ \\
\hline Fosfito C & 1500 & $104,1 \mathrm{a}$ & $682,2 \mathrm{~b}$ & $197,2 b$ \\
\hline Fosfito D & 1500 & $82,1 \mathrm{a}$ & $735,8 \mathrm{~b}$ & $201,4 b$ \\
\hline $\mathrm{ASM}^{2}$ & 50 & $96,8 \mathrm{a}$ & $535,0 \mathrm{~b}$ & $96,1 \mathrm{~b}$ \\
\hline Fungicida + Fosfito A & $300+1500$ & $44,8 \mathrm{a}$ & $53,7 \mathrm{a}$ & $20,9 \mathrm{a}$ \\
\hline Fungicida + Fosfito A & $400+1500$ & $50,2 \mathrm{a}$ & $16,3 \mathrm{a}$ & $10,5 \mathrm{a}$ \\
\hline Fungicida + ASM & $400+50$ & 59,7 a & $25,3 \mathrm{a}$ & $8,9 \mathrm{a}$ \\
\hline Fungicida & 500 & $39,0 \mathrm{a}$ & $14,6 \mathrm{a}$ & $8,7 \mathrm{a}$ \\
\hline C.V. $(\%)$ & & 10,17 & 14,12 & 13,92 \\
\hline
\end{tabular}

${ }^{1}$ Médias seguidas de letras distintas na mesma coluna diferem estatisticamente entre si pelo teste de Scott Knott a 5\% de probabilidade.

${ }^{2}$ ASM corresponde a acibenzolar-S-metil.

C.V.: Coeficiente de variação.

Os teores dos macronutrientes, principalmente de $\mathrm{P}$ e K, não foram alterados com as aplicações de fosfitos (Tabela 4). De acordo com valores desses nutrientes para a cultura de trigo (MALAVOLTA, 1992), os teores do nitrogênio, do fósforo e potássio estão adequados para a cultura. O teor de manganês com a aplicação do fosfito $\mathrm{D}$ foi alterado, ficando acima dos teores médios relacionados por Borkert, Pavan e Bataglia (2001). Isto sugere que aplicações com fosfito D podem elevar o teor de manganês, podendo ser nocivo para planta, já que o Mn é um micronutriente pouco móvel e durante o ciclo da cultura seus teores aumentam naturalmente, podendo resultar em deficiência de ferro devido a efeitos de competição por micronutrientes (EPSTEIN, 1972; LAMB, 1967). No entanto, no presente estudo não foram observados sintomas de toxicidade e de variações/reduções nos teores de $\mathrm{P}$ e $\mathrm{K}$, nem tampouco redução de produtividade. 
Tabela 4. Teores de nitrogênio $\left(\mathrm{N}^{*}\right)$, fósforo $(\mathrm{P})$, potássio $(\mathrm{K})$ e manganês $(\mathrm{Mn})$ presentes na folha bandeira de plantas de trigo, cultivar Avante, após aplicação de diferentes fontes de fosfitos e de indutor de resistência isolados e associados a fungicida, Safra 2006. Palmeira, PR.

\begin{tabular}{|c|c|c|c|c|c|}
\hline Tratamentos & Dose (mL p.a. ha' $\left.{ }^{-1}\right)$ & $\mathbf{N}\left(\mathbf{g ~ K \mathbf { g } ^ { - 1 }}\right)$ & $\mathbf{P}\left(\mathbf{g ~ K g ^ { - 1 }}\right)$ & $\mathbf{K}\left(\mathbf{g ~ K g}^{-1}\right)$ & $\operatorname{Mn}\left(\mathbf{m g ~ K g}{ }^{-1}\right)$ \\
\hline Testemunha & 1500 & $40,7^{\mathrm{ns}}$ & $3,4^{\mathrm{ns}}$ & 19,2 ns & $60,5 b^{1}$ \\
\hline Fosfito A & 1500 & 41,3 & 3,7 & 20,6 & $60,2 \mathrm{~b}$ \\
\hline Fosfito B & 1500 & 41,4 & 3,5 & 18,9 & $53,9 \mathrm{~b}$ \\
\hline Fosfito C & 1500 & 40,4 & 3,5 & 17,9 & $41,7 \mathrm{~b}$ \\
\hline Fosfito D & 50 & 40,6 & 3,7 & 19,0 & $157,4 \mathrm{a}$ \\
\hline $\mathrm{ASM}^{2}$ & $300+1500$ & 39,5 & 3,3 & 18,7 & $48,8 \mathrm{~b}$ \\
\hline Fungicida + Fosfito A & $400+1500$ & 40,2 & 3,5 & 20,4 & $53,1 \mathrm{~b}$ \\
\hline Fungicida + Fosfito A & $400+50$ & 41,5 & 3,4 & 20,0 & $71,6 \mathrm{~b}$ \\
\hline Fungicida + ASM & 500 & 40,7 & 3,4 & 18,6 & $53,1 \mathrm{~b}$ \\
\hline Fungicida & $66,5+2,5$ & 41,0 & 3,2 & 18,5 & $55,9 \mathrm{~b}$ \\
\hline C.V. $(\%)$ & & 3,1 & 4,9 & 9,1 & 24,18 \\
\hline
\end{tabular}

* Plantas coletadas no período da antese (antese completa).

${ }^{1}$ Médias seguidas de letras distintas na mesma coluna diferem estatisticamente entre si pelo teste de Scott Knott a $5 \%$ de probabilidade.

${ }^{2}$ ASM corresponde a acibenzolar-S-metil.

ns corresponde a não significativo.

C.V.: Coeficiente de variação.

Os maiores índices de área foliar foram obtidos nos tratamentos a base de piraclostrobina + epoxiconazole isolados ou em mistura com fosfito A e ASM (Tabela 5). Este resultado se repete nos resultados de PMS, peso hectolítrico e no rendimento, provavelmente devido ao fato do trigo ser altamente dependente da duração da área foliar e do índice de área foliar (HEINEMANN et al., 2006). Estes autores observaram uma alta correlação entre a produção de grãos e a eficiência de uso da radiação, índice de área foliar e biomassa na antese.

$\mathrm{Na}$ presença de genótipo suscetível e sob condições climáticas favoráveis ao desenvolvimento de epidemias, podem ocorrer graves epidemias principalmente das doenças que incidem na parte aérea como as ferrugens, o oídio e as manchas foliares. Barros, Castro e Patrício (2006) atribuem maiores perdas quando várias doenças incidem simultaneamente na lavoura. Durante a realização deste experimento, a ocorrência dessas doenças reduziram o potencial produtivo da cultivar (Tabela 2) que é superior aos $4000 \mathrm{~kg} \mathrm{ha}^{-1}$ (CULTIVARES OR, 2008). 
Tabela 5. Peso hectolítrico (PH), peso de mil sementes (PMS), produtividade (REN) e índice de área foliar (IAF*) da cultura de trigo, cultivar Avante, após aplicação de diferentes fontes de fosfitos e de indutor de resistência isolados e associados a fungicida, Safra 2006. Palmeira, PR.

\begin{tabular}{|c|c|c|c|c|c|}
\hline Tratamentos & Dose (mL p.a. ha $\left.{ }^{-1}\right)$ & PH (g) & PMS (g) & $\operatorname{REN}\left(\mathrm{Kg} \mathrm{ha}^{-1)}\right.$ & IAF \\
\hline Testemunha & ................. & $67,8 c^{1}$ & $29,7 \mathrm{~b}$ & $2425 \mathrm{~b}$ & $2,5 \mathrm{~b}$ \\
\hline Fosfito A & 1500 & $69,0 \mathrm{c}$ & $30,5 \mathrm{~b}$ & $2399 \mathrm{~b}$ & $2,7 \mathrm{~b}$ \\
\hline Fosfito B & 1500 & $67,0 \mathrm{c}$ & $28,3 \mathrm{~b}$ & $2095 \mathrm{~b}$ & $2,4 b$ \\
\hline Fosfito C & 1500 & $68,3 \mathrm{c}$ & $29,5 \mathrm{~b}$ & $2192 \mathrm{~b}$ & $2,5 \mathrm{~b}$ \\
\hline Fosfito D & 1500 & $68,3 \mathrm{c}$ & $29,8 \mathrm{~b}$ & $2345 \mathrm{~b}$ & $2,6 \mathrm{~b}$ \\
\hline $\mathrm{ASM}^{2}$ & 50 & $71,0 \mathrm{c}$ & $31,3 \mathrm{~b}$ & $2473 b$ & $2,7 b$ \\
\hline Fungicida + Fosfito A & $300+1500$ & $72,0 \mathrm{c}$ & $33,5 \mathrm{a}$ & $3121 \mathrm{a}$ & $3,2 \mathrm{a}$ \\
\hline Fungicida + Fosfito A & $400+1500$ & 73,9 a & $33,0 \mathrm{a}$ & 3283 a & $2,9 \mathrm{a}$ \\
\hline Fungicida + ASM & $400+50$ & $71,3 \mathrm{~b}$ & $33,6 \mathrm{a}$ & 3279 a & $3,0 \mathrm{a}$ \\
\hline Fungicida & 500 & $73,3 \mathrm{a}$ & $33,5 \mathrm{a}$ & $3369 \mathrm{a}$ & $3,0 \mathrm{a}$ \\
\hline C.V.(\%) & & 1,71 & 4,7 & 12,9 & 6,5 \\
\hline
\end{tabular}

*Mensurado na fase de formação de grão (grão em estádio inicial de massa).

${ }^{1}$ Médias seguidas de letras distintas na mesma coluna diferem estatisticamente entre si pelo teste de Tukey a 5\% de probabilidade.

${ }^{2} \mathrm{ASM}$ corresponde a acibenzolar-S-metil.

C.V.: Coeficiente de variação.

Somente o uso dos fosfitos não proporcionou aumento da produtividade (Tabela 5), nem de componentes do rendimento como o peso de mil sementes (PMS) e o peso hectolítrico (PH). Outros autores verificaram tendências semelhantes em outras culturas, como em laranjeiras, batatas, soja e em cebola (WRIGHT; PEÑA, 2002; JOHNSON; INGLIS; MILLER, 2004; NEVES, 2006; WORDELL FILHO, MARTINS; STADNIK, 2007). Nascimento et al. (2008) estudando o efeito de fontes de fosfitos e ASM no controle de doenças e produtividade do tomateiro obteve redução da mancha bacteriana pela formulação comercial Nutriphite $^{\circledR}$, mas não obteve ganho em produção. Segundo Mcdonald, Grant e Plaxton (2001) a participação do ácido fosforoso nas fases do metabolismo do fósforo é pequena, podendo ter pequeno ou nenhum efeito sobre a produtividade.

\section{Conclusão}

Os tratamentos com aplicação de fungicida de forma isolada ou associada ao fosfito A e a ASM proporcionaram controle da ferrugem da folha, do oídio e das manchas amarela e marrom do trigo. No entanto, somente a aplicação de diferentes fontes de fosfito não apresentou controle sobre essas doenças.

O fungicida de forma isolada e a combinação do fungicida com fosfito A e ASM proporcionaram aumento de produtividade.

$\mathrm{O}$ indutor de resistência acibenzolar-S-metil isoladamente ou em associação com fungicidas possui efeito sobre a ferrugem da folha e o oídio, além de proporcionar aumento na produtividade. 


\section{Referências}

BANZATTO, D. A. KRONKA, S. N. Experimentação agrícola. 4. ed. Jaboticabal: Funep, 2006. 237 p.

BARROS, B. C. Trigo/Brasil: o papel das doenças na baixa produtividade. Correio Agrícola, São Paulo, v. 2, p. 703-710, 1985.

BARROS, B. C.; CASTRO, J. L.; PATRÍCIO, F. R. A. Resposta de cultivares de trigo (Triticum aestivum L.) ao controle químico das principais doenças fúngicas da cultura. Summa Phytopathologica, Botucatu, v. 32, n. 3, p. 239-246, 2006.

BIZI, R. M.; GRIGOLETTI JUNIOR, A.; AUER, C. G.; MAY-DE MIO, L. L. Produtos alternativos no controle do oídio em mudas de eucalipto. Summa Phytopathologica., Botucatu, v. 34, n. 2, June 2008.

BORKERT, C. M.; PAVAN, M. A.; BATAGLIA, O. C. Disponibilidade e avaliação de elementos cationnicos: ferro e manganês. In: FERREIRA, M. E.; CRUZ, M. C. P.; VAN RAIJ, B.; ABREU, C. A. (Ed.). Micronutrientes e elementos tóxicos na agricultura. Jaboticabal: $\mathrm{CNPq} /$ FAPESP/POTAFOS, 2001. p. 151-186.

COSTA, M. J. N.; ZAMBOLIM, L.; RODRIGUES, F. A. Avaliação de produtos alternativos no controle da ferrugem do cafeeiro. Fitopatologia Brasileira, Brasília, v. 32, n. 2, p. 150-155, 2007.

CULTIVARES OR. Para quem busca lucratividade e segurança. 2008. Disponível em: <http://www. orsementes.com.br/prod.htm>. Acesso em: 29 maio 2008.

DALLAGNOL，L. J.; NAVARINI, L.; BALARDIN, R. S.; GOSENHEIMER, A.; MAFFINI, A. A. Dano das doenças foliares na cultura do arroz irrigado e eficiência de controle dos fungicidas. Revista Brasileira de Agrociência, Pelotas, v. 12, n. 3, p. 313-318, jul./set. 2006.

EMPRESA BRASILEIRA DE PESQUISA AGROPECUÁRIA - EMBRAPA. Centro Nacional de Pesquisa de Solos. Manual de análises de solos, plantas e fertilizantes. EMBRAPA, Brasília. 1999b. 370 p.

. Centro Nacional de Pesquisa de Solos. Sistema brasileiro de classificação de solos. EMBRAPA, São Paulo, 1999a. 412 p.

EPSTEIN, E. Mineral nutrition of plants: principles and perspectives. New York: John Wiley and Sons, Inc., 1972. $412 \mathrm{p}$.

GUEST, D. I.; GRANT, B. R. The complex action of phosphonates as antifungal agents. Biological Review, Cambridge, v. 66, n. 2, p. 159-187, 1991.
HEINEMANN, A. B.; STONE, L. F.; DIDONET, A. D.; TRINDADE, M. G.; SOARES, B. B.; MOREIRA, J. A. A.; CÁNOVAS, A. D. Eficiência de uso da radiação solar na produtividade do trigo decorrente da adubação nitrogenada. Revista Brasileira de Engenharia Agrícola Ambiental, Campina Grande, v. 10, n. 2, p. 352-356, 2006.

HETZLER, J.; EYAL, Z.; MEHTA, Y. R.; CAMPOS, L. A. C.; FEHRMANN, H.; KUSHNIR, U.; OREN, J. Z.; COHEN, L. Interaction between Cochliobolus sativus and wheat cultivars. In: SAUNDERS, D. A. (Ed.). International Conference On Wheat for Non-Traditional Warmer Areas. México:Cimmyt, 1991. p. 146-164.

JACKSON, T. J.; BURGESSA, T.; COLQUHOUNB, I.; HARDYA, G. E. STJ. Action of the fungicide phosphite on Eucaliptus marginata inoculated with Phytophthora cinnamomi. Plant Pathology, London, v. 49, p. 147-154, 2000.

JOHNSON, D. A.; INGLIS, D. A.; MILLER, J. S. Control of potato tuber rots caused by oomycetes with foliar applications of phosphorous acid. Plant Disease, St. Paul, v. 88, p. 1153-1159, 2004.

LAMB, C. A. Physiology. In: QUISENBERG, K. S.; REIZ, L. P. Wheat and wheat improvement. Madison, Wisconsin: American Society of Agronom, n. 13, 1967. p. 181-223.

MALAVOLTA, E. ABC da análise de solos e folhas: amostragem, interpretação e sugestões de adubação. São Paulo: Ceres, 1992. 124 p.

MARGOT, P.; HUGGENBERG, F.; AMREIN, J.; WEISS, B. A. CGA 279202: a new broad-spectrum strobilurin fungicide. Brighton: Brighton Crop Protection Conference on Plant Disease, 1998. p. 375-383.

McDONALD, A. E.; GRANT, B.; PLAXTON, W. C. Phosphite (phosphorous acid): Its relevance in the environment and agriculture and influence on plant phosphate starvation response. Journal Plant Nutrition, New York, v. 24, p. 1505-1519, 2001.

MEHTA, Y. R. Manejo integrado de las enfermedades del trigo. Santa Cruz de la Sierra: Imprenta Landivar, 1993, $314 \mathrm{p}$.

MEHTA. Y. R.; RIEDE, C. R.; CAMPOS, L. A. C.; KOHLI, M. M. Integrated management of major wheat diseases in Brazil: an example for the Southern Cone region of Latin America. Crop Protection, New York, v. 11, p. 517-524, 1992.

MOREIRA, L. M.; MAY-DE MIO, L. L.; VALDEBENITO-SANHUEZA, R. M.; LIMA, M. L. R. Z. C.; POSSAMAI, J. C. Controle em pós-colheita 
de Monilinia fructicola em pêssegos. Fitopatologia Brasileira, Brasília, v. 27, p. 395-398, 2002.

NASCIMENTO, A. R.; FERNANDES, P. M.; ROCHA, M. R.; SILVA, E. A.. Fontes de fosfito e acibenzolar-Smetil no controle de doenças e produtividade o tomateiro. Bioscience Journal, Uberlândia, v. 24, n. 1, p. 53-59, 2008.

NEVES, J. S. Influência da aplicação de fosfito de potássio na severidade da ferrugem asiática (Phakopsora pachyrhizi) da soja. 2006. Dissertação (Mestrado em Ciências Agrárias) - Universidade de Brasília, Brasília.

RAIJ, B. Van; CANTARELA, H.; QUAGGIO, J. A.; FURLANI, A. M. C. Recomendações de adubação e calagem para o Estado de São Paulo. 2. ed. Campinas: IAC, 1997. 285 p. (Boletim técnico, 100).

REIS, E. M.; CASA, R. T.; FORCELINI, C. A. Relação entre a severidade e a incidência da ferrugem da folha do trigo, causada por Puccinia triticina f. sp. tritici. Fitopatologia Brasileira, Brasília, v. 21, n. 3, p. 369-372, 1996.

REIS, E. M.; CASA, R. T.; MEDEIROS, C. A. Diagnose, patometria e controle de doenças de cereais de inverno. Londrina: ES Comunicação S/C Ltda, 2001. 94 p.

REIS, E. M.; CASA, R. T. Doenças dos cereais de inverno: diagnose, patometria e controle de doenças de cereais de inverno. Lages: Graphel, 2007. 176 p.

REUVENI, M. Post-infection applications of $\mathrm{K}_{3} \mathrm{PO}_{3}$, phosphorous acid and imethomorph inhibit development of downy mildew caused by Plasmopara viticola on grapevines. Journal of Small Fruit \& Viticulture, Baton Rouge, v. 5, n. 22, p. 27-38, 1997.

SHANER, G.; FINNEY, R. E. The effect of nitrogen fertilization on the expression of slow-mildewing resistance in knox wheat. Phytopathology, St. Paul, v. 67, n. 8, p. 1051-1056, 1977.

SÔNEGO, O. R.; GARRIDO, L. R.; CZERMAINSKI, A. B. C. Avaliação do fosfito de potássio no controle do míldio da videira. Bento Gonçalves: Embrapa Uva e Vinho, 2003. 16 p. (Boletim de Pesquisa e Desenvolvimento, 11).

VITTI, G. C.; LUZ, P. H. C.; OTTO, R.; QUEIROS, F. E. C.; PACKER, L. A. Utilização de fosfitos em canade-açúcar. In: SIMPÓSIO DE TECNOLOGIA DE PRODUÇÃO DE CANA-DE-AÇÚCAR, Piracicaba. Anais... Piracicaba: GAPE-GELQ-ESALQ/USP, 2005. p. 17.

WORDELL FILHO, J. A.; MARTINS, D. A.; STADNIK, M. J. Aplicação foliar de tratamentos para o controle do míldio e da podridão-de-escamas de bulbos de cebola.
Horticultura Brasileira, Campinas, v. 25, n. 4, p. 544549, 2007.

WRIGHT, C. G.; PEÑA. M. Foliar applications of lobiuret urea and potassium phosphite to navel orange trees. 2002. Disponível em: <http://ag.arizona.edu/pubs/ crops/az1303/az1303-3.pdf>. Acesso em: 21 maio 2008.

ZADOKS, J. C.; CHANG, T. T.; KONZAK, C. F. A decimal code for the growth stages of cereals. Weed Research, Oxford, v. 14, n. 6, p. 415-421, 1974. 